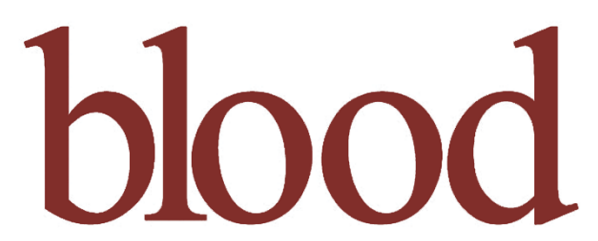

2005 106: 3768-3776

Prepublished online Aug 16, 2005;

doi:10.1182/blood-2005-04-1746

\title{
Relationship between FLT3 mutation status, biologic characteristics, and response to targeted therapy in acute promyelocytic leukemia
}

Rosemary E. Gale, Robert Hills, Arnold R. Pizzey, Panagiotis D. Kottaridis, David Swirsky, Amanda F. Gilkes, Elizabeth Nugent, Kenneth I. Mills, Keith Wheatley, Ellen Solomon, Alan K. Burnett, David C. Linch, David Grimwade and for the NCRI Adult Leukaemia Working Party

Updated information and services can be found at:

http://bloodjournal.hematologylibrary.org/cgi/content/full/106/12/3768

Articles on similar topics may be found in the following Blood collections:

Oncogenes and Tumor Suppressors (796 articles)

Clinical Trials and Observations (2398 articles)

Neoplasia (4075 articles)

Information about reproducing this article in parts or in its entirety may be found online at:

http://bloodjournal.hematologylibrary.org/misc/rights.dtl\#repub_requests

Information about ordering reprints may be found online at:

http://bloodjournal.hematologylibrary.org/misc/rights.dtl\#reprints

Information about subscriptions and ASH membership may be found online at:

http://bloodjournal.hematologylibrary.org/subscriptions/index.dtl

Blood (print ISSN 0006-4971, online ISSN 1528-0020), is published semimonthly by the American Society of Hematology, $1900 \mathrm{M} \mathrm{St}$, NW, Suite 200, Washington DC 20036.

Copyright 2007 by The American Society of Hematology; all rights reserved.

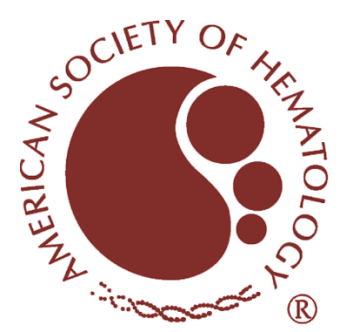




\title{
Relationship between FLT3 mutation status, biologic characteristics, and response to targeted therapy in acute promyelocytic leukemia
}

\author{
Rosemary E. Gale, Robert Hills, Arnold R. Pizzey, Panagiotis D. Kottaridis, David Swirsky, Amanda F. Gilkes, Elizabeth Nugent, \\ Kenneth I. Mills, Keith Wheatley, Ellen Solomon, Alan K. Burnett, David C. Linch, and David Grimwade, \\ for the NCRI Adult Leukaemia Working Party
}

\begin{abstract}
The prognostic significance of FLT3 mutations in acute promyelocytic leukemia (APL) is not firmly established and is of particular interest given the opportunities for targeted therapies using FLT3 inhibitors. We studied 203 patients with PMLRARA-positive APL; $43 \%$ of the patients had an FLT3 mutation (65 internal tandem duplications [ITDs], 19 D835/I836, 4 ITD+D835/1836). Both mutations were associated with higher white blood cell (WBC) count at presentation; $75 \%$ of the patients with WBC counts of $10 \times 10 \% / \mathrm{L}$ or
\end{abstract}

greater had mutant FLT3. FLT3/ITDs were correlated with M3v subtype $(P<.001)$, bcr3 $P M L$ breakpoint $(P<.001)$, and expression of reciprocal $R A R A-P M L$ transcripts $(P=.01)$. Microarray analysis revealed differences in expression profiles among patients with FLT3/ITD, D835/I836, and wild-type FLT3. Patients with mutant FLT3 had a higher rate of induction death ( $19 \%$ vs $9 \%$; $P=.04$, but no significant difference in relapse risk $(28 \%$ vs $23 \%$; $P=.5)$ or overall survival $(59 \%$ vs $67 \%$; $P=.2)$ at 5 years. In in vitro differentia- tion assays using primary APL blasts $(n=6)$, the FLT3 inhibitor CEP-701 had a greater effect on cell survival/proliferation in FLT3/ITD ${ }^{+}$cells, but this inhibition was reduced in the presence of ATRA. Furthermore, in the presence of CEP-701, ATRA-induced differentiation was reduced in FLT3/ITD+ cells. These data carry implications for the use of FLT3 inhibitors as frontline therapy for APL. (Blood. 2005; 106:3768-3776)

() 2005 by The American Society of Hematology

\section{Introduction}

Most cases of acute promyelocytic leukemia (APL) are characterized by $\mathrm{t}(15 ; 17)(\mathrm{q} 22 ; \mathrm{q} 21)$ leading to formation of the promyelocytic leukemia-retinoic acid receptor $\alpha$ (PML-RARA) fusion protein. ${ }^{1}$ PML-RARA plays a critical role in determining disease phenotype, mediating the characteristic differentiation block through the repression of genes implicated in myelopoiesis, which is overcome by pharmacologic levels of retinoic acid. ${ }^{1}$ However, evidence derived largely from transgenic mouse models has suggested that PML-RARA is insufficient for leukemogenesis, ${ }^{2,3}$ although the precise nature of the cooperating events implicated in generating the full disease phenotype remains uncertain. A number of potential candidates have been proposed to play a role in this process. These include the reciprocal fusion gene product RARAPML, which is expressed in approximately $75 \%$ of patients ${ }^{4-6}$ and has been postulated to contribute to leukemogenesis by promoting genomic instability, thereby predisposing to the acquisition of additional oncogenic lesions. ${ }^{7}$ There has also been considerable interest in the potential role of activating mutations of genes encoding receptor tyrosine kinases (RTKs), which commonly

From the Department of Haematology, University College London Hospitals, London; Clinical Trials Unit, University of Birmingham; Haematological Malignancy Diagnosis Service, Department of Haematology, Leeds General Infirmary; Department of Haematology, University of Cardiff, Wales; and Department of Medical and Molecular Genetics, King's College London, United Kingdom.

Submitted April 29, 2005; accepted July 23, 2005. Prepublished online as Blood First Edition Paper, August 16, 2005; DOI 10.1182/blood-2005-04-1746.

A complete list of the members of the National Cancer Research Institute (NCRI) Adult Leukaemia Working Party appears in "Appendix."

Supported by the Leukaemia Research Fund of Great Britain (D.G., P.D.K., E.N.). The DNA/RNA tissue bank is supported by the Kay Kendall Leukaemia Fund, the Leukaemia Research Fund of Great Britain, and the UK Medical Research Council. accompany acute myelocytic leukemia (AML)-associated translocations including $\mathrm{t}(15 ; 17)$, giving rise to the proposition that they could provide a common class of cooperating mutation in the development of the disease. ${ }^{8}$

Fms-like tyrosine kinase 3 (FLT3) is an RTK expressed on hematopoietic progenitors. Mutation of the FLT3 gene is common in AML. ${ }^{9-12}$ Numerous mutations have been identified. The majority, present in approximately $25 \%$ of patients, are internal tandem duplications (ITDs) that lead to in-frame insertions within the juxtamembrane region of the receptor. Less frequent are mutations involving the region encoding the activation loop, which most commonly affect codons aspartate 835 and isoleucine 836 (D835/ I836) and have been reported in approximately $8 \%$ of patients with AML. ${ }^{9-12}$ In vitro studies have revealed that both classes of mutation lead to constitutive activation of the receptor. However, whereas most large studies of AML have found the presence of an ITD to be an adverse prognostic indicator predicting for higher incidence of relapse, the significance and biologic characteristics of FLT3 activation loop mutations remain uncertain and, for reasons
R.E.G., A.R.P., P.D.K., A.F.G., E.N., and D.G. performed experimental analysis. R.H. and K.I.M. performed data analysis. K.W. contributed statistical advice and interpretation. D.S. performed morphologic review. A.K.B., D.C.L., and E.S. contributed to the design of the study and the interpretation of the data. R.E.G. and D.G wrote the manuscript, with contributions from the other authors as appropriate.

The online version of the article contains a data supplement.

Reprints: David Grimwade, Department of Medical and Molecular Genetics, King's College London, 8th fl, Guy's Tower, Guy's Hospital, London SE1 9RT, United Kingdom; e-mail: david.grimwade@genetics.kcl.ac.uk.

The publication costs of this article were defrayed in part by page charge payment. Therefore, and solely to indicate this fact, this article is hereby marked "advertisement" in accordance with 18 U.S.C. section 1734.

(C) 2005 by The American Society of Hematology 
that are unclear, they do not appear to predict for poor outcome. ${ }^{10-12}$ This difference in prognostic impact may reflect the influence of these mutations on the range and the extent of activation of downstream signal transduction pathways.

Intriguingly, the frequency of FLT3 ITDs varies dramatically across cytogenetically and molecularly defined subsets of AML, and they are particularly prevalent in patients with $t(15 ; 17) .{ }^{13-19}$ Although previous studies considering patients with APL have highlighted an association between ITDs and elevated presenting white blood cell (WBC) count, hypogranular variant (M3v) morphology, and the short (bcr3) isoform of PML-RARA, ${ }^{15,17,19-26}$ the prognostic significance of FLT3 mutations in APL has not been firmly established. ${ }^{17,20-26}$ This is important because it has a potential bearing on treatment stratification in this disease and is highly pertinent given the recent clinical interest in drugs targeting FLT3. In vitro studies of small-molecule FLT3 inhibitors have shown that they can suppress proliferation in ITD-expressing cell lines and in primary AML blast cells $\mathrm{s}^{27-34}$ and can prolong survival in mouse models of ITD-induced disease. ${ }^{35-38}$ Furthermore, one agent (SU11657) has shown promising results in combination with all-trans retinoic acid (ATRA) in an APL mouse model, achieving rapid elimination of blasts coexpressing PML-RARA and mutant FLT3.${ }^{37}$ Phase $1 / 2$ trials of FLT3 inhibitors as single agents have led to partial hematologic responses in a proportion of patients with refractory, relapsed, or poor-risk AML. ${ }^{39-44}$ However, studies with the indolocarbazole alkaloid CEP-701 found that a clinical response was only observed in patients demonstrating in vitro sensitivity to the agent in a cytotoxicity assay and greater than $85 \%$ in vivo inhibition of FLT3 autophosphorylation. ${ }^{43}$ Efficacy may also depend on leukemic subtype; to date, APL patients have generally been excluded from these trials, although results from the mouse models lend support to the hypothesis that APL is a good candidate for the evaluation of FLT3 inhibitors. ${ }^{37}$

To address these issues we have studied the impact of FLT3 mutations on disease characteristics and clinical outcome in a large cohort of APL patients treated in the United Kingdom Medical Research Council (MRC) AML10 and AML12 trials. In addition, to further investigate the rationale for using FLT3 inhibitors as frontline therapy in APL, we have assessed the in vitro effect of CEP-701 in the presence or absence of ATRA in primary APL blasts.

\section{Patients, materials, and methods}

\section{Patients and therapy}

The study group consisted of 203 consecutive patients with $\mathrm{t}(15 ; 17) / P M L$ $R A R A$-associated APL (median age, 37 years; range, 1-60 years) with available diagnostic DNA or RNA treated in the UK MRC AML10 and AML12 trials. ${ }^{45}$ Twenty patients were treated in the AML10 trial before the availability of ATRA; 110 patients were entered into the MRC ATRA trial ${ }^{45}$ in which patients were randomly assigned to receive a short 5-day course of ATRA before induction chemotherapy $(n=57)$ or an extended course of ATRA begun simultaneously with induction chemotherapy and continued until achievement of complete remission $(\mathrm{CR})(\mathrm{n}=53)$. In the remaining patients, ATRA was not randomized, but in most it was given according to current clinical practice (ie, an extended course as per the MRC ATRA trial). Overall, 17 patients treated in the AML10 or AML12 protocols underwent transplantation in first CR ( 9 allogeneic, 8 autologous). This study was approved by the Multi-center Research Ethics Committee (MREC) for Wales. Informed consent was provided according to the Declaration of Helsinki.

\section{Confirmation of diagnosis of APL}

Cytogenetic analysis/detection of PML-RARA fusion. All patients were confirmed by conventional cytogenetics to have $t(15 ; 17)^{46}$ or by nested reverse transcriptase-polymerase chain reaction (RT-PCR) to have the PML-RARA fusion transcript. ${ }^{6}$ Cytogenetic results were available in 187 patients. RT-PCR to detect $P M L-R A R A$ and RARA-PML transcripts and to determine $P M L$ breakpoints was undertaken in 176 patients. ${ }^{6}$ In view of the relative rarity of the bcr2 subtype $(n=13)$, results for patients with bcr1 and bcr2 were combined for further analyses.

Morphologic review. Diagnostic slides were available for central morphologic review in 104 patients and were classified into hypergranular (M3) and hypogranular/microgranular variant (M3v) forms according to previously described criteria. ${ }^{47}$ In addition, patients with the basophilic variant of APL (M3B) were distinguished. ${ }^{48,49}$

\section{Determination of FLT3 mutation status}

The juxtamembrane domain of the FLT3 gene was amplified from DNA or cDNA as previously described ${ }^{13}$; any patient with an additional higher molecular weight band was considered to be positive for an ITD $\left(\operatorname{ITD}^{+}\right)$, irrespective of the size of the band or the relative level of mutant. The presence and quantification of an ITD was confirmed by PCR amplification with a fluorescently labeled primer followed by fragment analysis on the CEQ 8000 DNA Genetic Analysis System (Beckman Coulter, High Wycombe, United Kingdom). Point mutations at codon D835 or I836 were detected using a modified method of the original PCR $+E c o$ RV digestion procedure ${ }^{50}$ One PCR primer was as previously reported $\left(17 \mathrm{~F}^{50}\right)$, and the other primer was modified to introduce an EcoRV digestion site that allowed discrimination of undigested products from those cut once (mutant, D835 ${ }^{+}$) or twice (wild type [WT]). For DNA (mismatch primer 5'-CAGTGAGTGCAGTTGTTTACCATGATATCG-3'; mismatch underlined), 35 cycles of amplification were performed with an annealing temperature of $63^{\circ} \mathrm{C} . E c o R V$ digestion of the 180-bp product gave bands of $154+26 \mathrm{bp}$ for mutant alleles and $90+64+26$ bp for WT alleles. For cDNA (mismatch primer, 5'-CACAGTAATATTCCATATGACCAGATATC- $3^{\prime}$ ), the annealing temperature was $60^{\circ} \mathrm{C}$, the product was $199 \mathrm{bp}$, and the digested bands were $173+26 \mathrm{bp}$ for mutant alleles and $105+68+26 \mathrm{bp}$ for WT alleles.

\section{Gene chip analysis}

Total RNA was extracted using TRIzol reagent (Invitrogen, Paisley, United Kingdom) and was subjected to qualitative and quantitative analysis by Agilent Bioanalyzer 2100 (Agilent Technologies, South Queensferry, United Kingdom). Total RNA $(7.5 \mu \mathrm{g})$ was converted to double-stranded cDNA using SuperScript II reverse transcriptase (Invitrogen) and T7-Oligo(dT) promoter primer (Affymetrix, High Wycombe, United Kingdom) and then cleaned, precipitated, and transcribed into biotin-labeled cRNA using the Enzo BioArray High Yield RNA Transcript Labelling Kit (Affymetrix). The cRNA was cleaned using ChromaSpin columns (BD Biosciences Clontech, Oxford, United Kingdom), fragmented, and hybridized overnight to a Human U133A GeneChip (Affymetrix). GeneChips were then washed, stained, and scanned. Initial analysis used Affymetrix Microarray Suite software, version 5.0 (MAS5.0); further analysis used GeneSpring 6.2 software (Affymetrix). Data were normalized and filtered to remove genes that were flagged as absent in all samples according to the MAS5.0 software. Three tests were performed to identify genes that correlated with FLT3 status - an analysis of variance (ANOVA) parametric test where the variances were not assumed to be equal, an association test for each gene using Fisher exact test for association between expression level and class membership, and a significance analysis of microarray (SAM) ${ }^{51}$

\section{In vitro differentiation assays}

The PML-RARA ${ }^{+}$APL cell line NB4 ${ }^{52}$ was cultured in RPMI 1640 supplemented with $10 \%$ fetal calf serum (FCS) and penicillin/streptomycin. Mononuclear cells (MNCs) from bone marrow $(n=2)$ or peripheral blood $(n=4)$ of patients at presentation with PML-RARA ${ }^{+}$APL were obtained by standard Ficoll-Hypaque density gradient centrifugation. All patient samples were used fresh within 24 hours of collection. Cells were resuspended in RPMI/10\% FCS at a density of $5 \times 10^{4} / \mathrm{mL}(\mathrm{NB} 4)$ or $1 \times 10^{6} / \mathrm{mL}$ (MNCs) and were cultured in the 
presence of nothing [control cells], $1 \mu$ M ATRA(Sigma Chemical, Poole, United Kingdom), $50 \mathrm{nM}$ CEP-701 (a kind gift from Cephalon, West Chester, PA), or 1 $\mu \mathrm{M}$ ATRA $+50 \mathrm{nM} \mathrm{CEP}-701$. The final concentration of dimethylsulfoxide was less than $0.005 \%$. After 2 to 5 days of incubation at $37^{\circ} \mathrm{C}$ in $5 \% \mathrm{CO}_{2}$, cells were counted and assessed for viability (trypan blue exclusion) and apoptosis (flow cytometric analysis of fluorescein isothiocyanate [FITC]-conjugated annexin V expression and propidium iodide [PI] exclusion). Cell proliferation was assessed in triplicate on $1 \times 10^{3} \mathrm{NB} 4$ cells or $1 \times 10^{5}$ leukemic blasts using the CellTiter 96 AQueous One Solution Cell Proliferation (MTS) Assay (Promega, Southampton, United Kingdom), and values were normalized to those obtained for the control cells. The degree of differentiation was determined by immunophenotyping and flow cytometric analysis using phycoerythrin (PE)-conjugated antiCD11b (DakoCytomation, Ely, United Kingdom) and corrected for isotypespecific control antibody binding. Acquisition of respiratory burst activity was assessed by the production of hydrogen peroxide using $2^{\prime} 7^{\prime}$ dichlorofluorescein diacetate (DCFDA; Molecular Probes, Eugene, OR) as previously described. ${ }^{53}$ Aliquots of $2 \times 10^{5}$ cells were loaded with $10 \mu \mathrm{M}$ DCFDA for 15 minutes at $37^{\circ} \mathrm{C}$, then incubated in the presence or absence of $1 \mu \mathrm{g} / \mathrm{mL}$ 12-0-tetradecanoylphorbol 13-acetate (TPA; Sigma Chemical) for 5 minutes at $37^{\circ} \mathrm{C}$, and the relative percentage of positive cells was determined by flow cytometry.

\section{Definitions of end points}

A normocellular bone marrow aspirate containing less than 5\% blasts and showing evidence of normal maturation of other marrow elements was the criterion for the achievement of complete remission (CR). Full recovery of normal peripheral blood counts was not required to define $\mathrm{CR}$, although at least $90 \%$ of patients considered to be in CR according to the protocol definition also satisfied National Cancer Institute criteria. ${ }^{54}$ Remission failures were classified by the referring clinician either as caused by induction death (ID) related to treatment, hypoplasia, or both, or by resistant disease (RD) related to the failure of therapy to eliminate the disease (including partial remissions with 5\%-15\% blasts). Overall survival (OS) refers to the proportion of patients still alive at a given time after entry to the trial, and relapse risk (RR) is the cumulative probability of relapse (ie, censoring at death in $\mathrm{CR}$ ).

\section{Statistical methods}

Standard statistical techniques were used to relate FLT3 status to clinical outcome. Survival and relapse data were analyzed using log-rank tests or, for model building, proportional hazards (Cox) regression models. Fiveyear percentages were obtained using Kaplan-Meier estimates. Two-way associations between variables were assessed using the Fisher exact test or the Mantel-Haenszel test for trend for associations between categorical and ordinal data, and standard $t$ tests and Wilcoxon rank-sum tests were used for scale variables. Model building for CR was performed using logistic regression. All models were constructed using forward selection and an entry probability of .05 . Patients were censored for follow-up on April 1, 2004; date of death or follow-up to April 1, 2004, was available for $88 \%$ of patients. Patients who were lost to follow-up were censored at the date they were last known to be alive. Median follow-up was 7.2 years (range, 1.8-14.9 years).

\section{Results}

\section{Relationship between FLT3 mutation status and disease characteristics}

FLT3 mutation status (ITD and D835/I836) was determined in 203 patients with PML-RARA-associated APL. In total, 88 (43\%) of 203 patients had mutations-65 (32\%) had ITD mutations, $19(9 \%)$ had D835/I836 mutations, and $4(2 \%)$ had both mutations. The presence of either type of mutation was associated with higher presenting WBC count (Table 1). Considering the relationship between WBC count and FLT3 status, $31 \%$ of patients with WBC counts lower than $10 \times 10^{9} / \mathrm{L}$ and $75 \%$ with WBC counts of $10 \times 10^{9} / \mathrm{L}$ or greater had mutant FLT3. The presence of an ITD correlated with $\mathrm{M} 3 \mathrm{v}$ subtype $(P<.001)$, bcr3 $P M L$ breakpoint $(P<.001)$, and reciprocal $R A R A-P M L$ transcript expression $(P=.01)$; no correlations were observed with the presence of a D835/I836 mutation (Table 1). However, given the small numbers of patients with D835/I836 mutations, it is impossible to rule out a smaller but still potentially relevant effect of this mutation on morphology or breakpoint. There was no evidence that FLT3 mutation correlated with the presence of additional cytogenetic abnormalities; indeed, patients with such changes had lower frequency of FLT3 mutation $(P=.002)$.

Table 1. Relationship between FLT3 mutation status and disease characteristics in 203 APL patients

\begin{tabular}{|c|c|c|c|c|c|c|}
\hline & Total & WT & ITD* & $P$ & D835/1836 only & $P$ \\
\hline No. patients & 203 & 115 & 69 & & 19 & \\
\hline Median age, y (range) & $37(1-60)$ & $36(7-60)$ & $41(1-59)$ & .4 & $32(4-52)$ & .5 \\
\hline Median WBC count, $\times 10^{9} / \mathrm{L}$ (range) & $3.5(0.2-195)$ & $2.2(0.3-140)$ & $8.9(0.2-195)$ & $<.001$ & $8.9(0.8-44.7)$ & .001 \\
\hline Median platelet count, $\times 10^{9} / \mathrm{L}$ (range) & $23(3-153)$ & $23.5(7-153)$ & $23(3-99)$ & 3 & $23(7-83)$ & .6 \\
\hline Morphologic features, no. (\%) & & & & $<.001$ & & .9 \\
\hline M3 & $63(61)$ & $39(75)$ & $15(38)$ & & $9(69)$ & \\
\hline M3v & $37(36)$ & $10(19)$ & $24(62)$ & & $3(23)$ & \\
\hline M3B & $4(4)$ & $3(6)$ & $0(0)$ & & $1(8)$ & \\
\hline Unknown & 99 & 63 & 30 & & 6 & \\
\hline Cytogenetics, no. (\%) & & & & .002 & & .5 \\
\hline$t(15 ; 17)$ alone & $124(66)$ & $60(56)$ & $52(83)$ & & $12(71)$ & \\
\hline $\mathrm{t}(15 ; 17)+$ other abnormalities & $49(26)$ & $36(33)$ & $9(14)$ & & $4(24)$ & \\
\hline PML-RARA+, not $(15 ; 17)$ & $14(8)$ & $11(10)$ & $2(3)$ & & $1(6)$ & \\
\hline No cytogenetics & 16 & 8 & 6 & & 2 & \\
\hline PML breakpoint, no. (\%) & & & & $<.001$ & & .6 \\
\hline Bcr $1 / 2$ & $115(65)$ & $77(76)$ & $26(45)$ & & $12(71)$ & \\
\hline Bcr 3 & $61(35)$ & $24(24)$ & $32(55)$ & & $5(29)$ & \\
\hline Unknown & 27 & 14 & 11 & & 2 & \\
\hline RARA-PML expression, no. (\%) & & & & .01 & & $>.999$ \\
\hline Negative & $42(24)$ & $30(30)$ & $7(12)$ & & $5(29)$ & \\
\hline Positive & $134(76)$ & $71(70)$ & $51(85)$ & & $12(71)$ & \\
\hline Unknown & 27 & 14 & 11 & & 2 & \\
\hline
\end{tabular}

Percentages may not add up to $100 \%$ because of rounding. $P$ values were determined by $t$ test for age and by Wilcoxon rank sum test for WBC and platelet counts. All comparisons are made with the WT group.

${ }^{*}$ Includes 4 patients with coexistent FLT3 ITD and D835/I836. 
From www.bloodjournal.org at UCL Library Services on August 20, 2008. For personal use only.

\section{Impact of FLT3 mutation status on outcome}

There was a significantly higher ID rate in patients with the FLT3 mutation (odds ratio [OR] 2.50; $95 \%$ confidence interval $(95 \% \mathrm{CI}$ ), 1.11-5.65; $P=.04)$, underlying the suggestion of a lower $\mathrm{CR}$ rate (OR, 1.99; 95\% CI, 0.94-4.22; $P=.09$ ) (Table 2). However, no independent effect of FLT3 mutation was observed after adjusting for WBC count (ID: OR, 1.82; 95\% CI, 0.71-4.63; $P=.2$; CR: OR, $1.65 ; 95 \% \mathrm{CI}, 0.71-3.85 ; P=.2$ ). Of the 17 deaths in patients with mutant FLT3, 8 resulted from hemorrhage, 4 from sepsis, 1 from cardiac arrhythmia, and 4 from retinoic acid syndrome. Eight deaths occurred within 4 days of trial entry. There was no significant difference in RR according to FLT3 mutation status (ITD vs wild-type [WT], $P=.5$; D835/I836 vs WT, $P=.9$; either mutation vs WT, $P=.5$ ) (Table 2; Figure 1A). There was borderline significantly worse OS in patients with a D835/I836 mutation $(P=.05)$, but not in those with an $\operatorname{ITD}(P=.5)$ or in all patients with a mutation taken collectively $(P=.2)$ (Table 2; Figure 1B), which in part is likely to reflect the relatively high rate of ID in the former group. Multivariate regression analysis showed the presenting WBC count to be the most significant prognostic factor $(P=.001, P<.001$, and $P<.001$ for ID, RR, and OS, respectively) and found no evidence that either age or ATRA randomization influenced the effect of an FLT3 mutation on outcome among the APL group.

To determine whether the impact of an ITD on outcome in APL differs from that in non-APL AML patients younger than 60 years of age, a stratified analysis was undertaken comparing RR for the current group of 170 APL patients with that for 861 confirmed non-APL patients (median age, 42 years) also treated in the MRC AML10 and AML12 trials. No significant heterogeneity between the 2 groups was found (Figure 1C).

\section{APL with FLT3 ITD and D835/1836 mutations have different gene expression profiles}

RNA from bone marrow samples of 26 APL patients was analyzed using U133A GeneChips (Affymetrix); 15 had WT FLT3, 5 were positive for ITD, 5 were positive for D835, and 1 was positive for both mutations. After normalization and filtering, 13352 genes remained. ANOVA on the basis of FLT3 status identified 1008 differentially expressed genes; 980 of these genes were also identified by the association test; 83 probe sets overlapped between the SAM and ANOVA analyses (Tables S1 and S2, available on the Blood website; see the Supplemental Tables link at the top of the
A

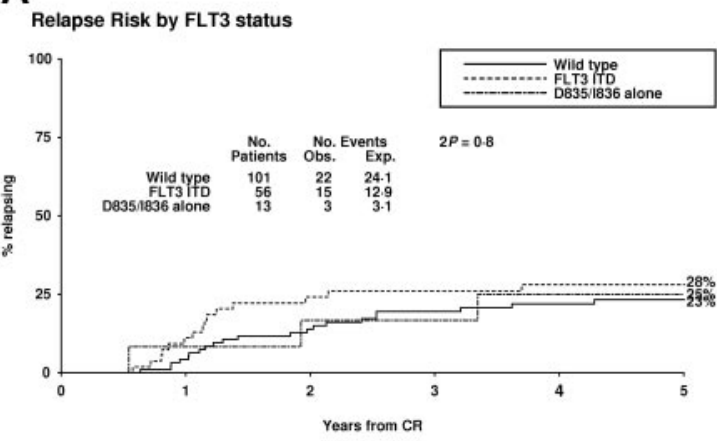

B Overall Survival by FLT3 status

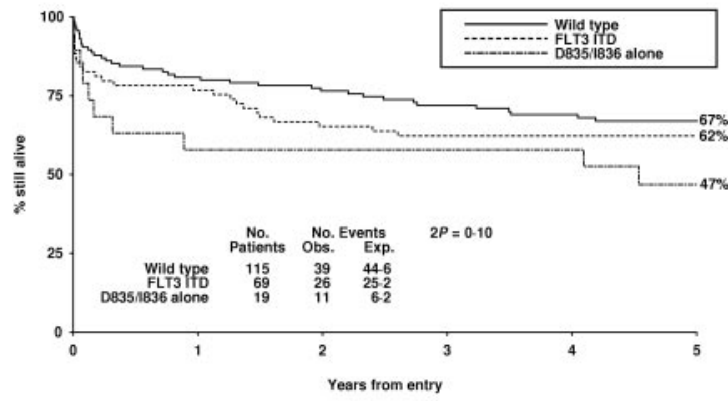

C Relapse Rate by FLT3 ITD Status Stratified by APL

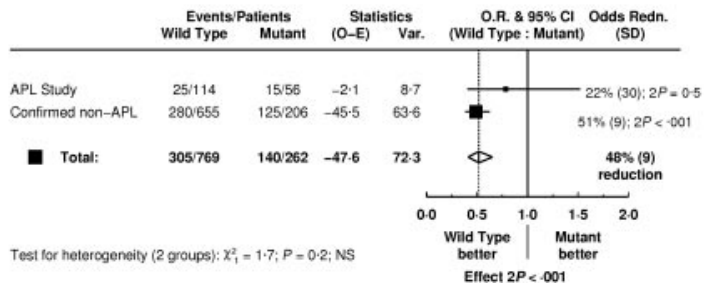

Figure 1. Impact of FLT3 mutation status on outcome in APL patients. (A) Relapse risk. (B) Overall survival. (C) Relapse risk in patients with APL and non-APLAML.

online article). Hierarchical cluster analysis using these 83 probe sets, which represented 78 different genes, identified 2 major clusters (Figure 2). One cluster contained 10 samples that divided into 2 subclusters, one with $5 \mathrm{ITD}^{+}$samples and the other with 4

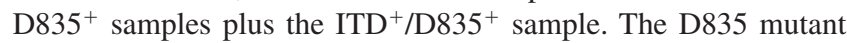

Table 2. Prognostic significance of FLT3 mutation status in APL

\begin{tabular}{|c|c|c|c|c|c|c|c|c|}
\hline & Total & WT & ITD* & $P$ & D835/1836 only & $P$ & Either mutant & $P$ \\
\hline No. & 203 & 115 & 69 & & 19 & & 88 & \\
\hline ID, \% & 13 & 9 & 17 & .10 & 26 & .04 & 19 & .04 \\
\hline OR $(95 \% \mathrm{Cl})$ & - & - & $2.27(0.91-5.68)$ & & $5.81(1.25-26.9)$ & & $2.50(1.11-5.65)$ & \\
\hline RD, \% & 2 & 3 & 1 & .7 & 5 & .5 & 2 & .7 \\
\hline OR $(95 \% \mathrm{Cl})$ & - & - & $0.47(0.07-2.91)$ & & $1.64(0.13-21.0)$ & & $0.66(0.13-3.38)$ & \\
\hline CR, \% & 84 & 88 & 81 & .3 & 68 & .04 & 78 & .09 \\
\hline OR $(95 \% \mathrm{Cl})$ & - & - & $1.70(0.73-3.94)$ & & $4.56(1.17-17.7)$ & & $1.99(0.94-4.22)$ & \\
\hline \multicolumn{9}{|l|}{ Outcome at $5 \mathrm{y}$} \\
\hline RR at $5 \mathrm{y}, \%$ & 25 & 23 & 28 & .5 & 25 & .9 & 28 & .5 \\
\hline OR $(95 \% \mathrm{Cl})$ & - & - & $1.29(0.65-2.53)$ & & $1.06(0.31-3.62)$ & & $1.24(0.66-2.34)$ & \\
\hline OS at $5 y, \%$ & 64 & 67 & 62 & .5 & 47 & .05 & 59 & .2 \\
\hline OR $(95 \% \mathrm{Cl})$ & - & - & $1.19(0.72-1.98)$ & & $2.55(1.09-5.98)$ & & $1.36(0.86-2.15)$ & \\
\hline
\end{tabular}

Percentages may not add up to $100 \%$ because of rounding. All comparisons are made with the WT group.

ID indicates induction death; $\mathrm{OR}$, odds ratio; $\mathrm{Cl}$, confidence interval; $\mathrm{RD}$, resistant disease; $\mathrm{CR}$, complete remission; $\mathrm{RR}$, relapse risk; OS, overall survival; - , not applicable.

${ }^{*}$ Includes 4 patients with coexistent FLT3 ITD and D835/I836 mutation. 


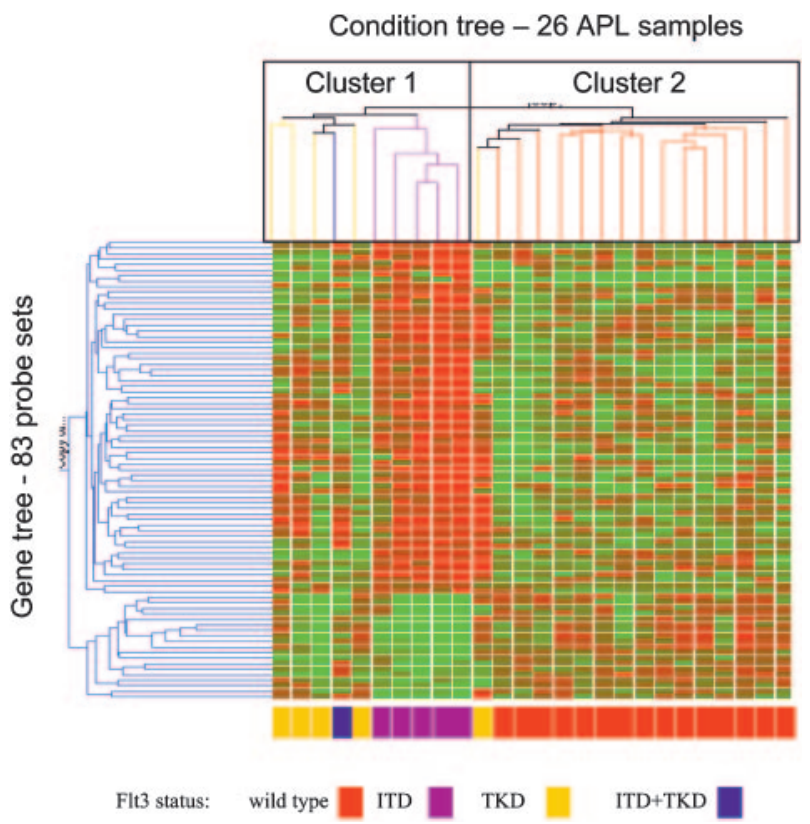

Figure 2. Hierarchical clustering of genes according to FLT3 status in 26 APL samples. The 83 probe sets shown were identified by SAM analysis and represent 78 different genes.

was the predominant mutation in the latter sample. The second major cluster contained all WT FLT3 samples and a single high-level mutant $\mathrm{D} 835^{+}$sample. There was no correlation between cluster type and WBC count (greater or less than $10 \times 10^{9} \%$ $\mathrm{L}$ ), morphologic features, $P M L$ breakpoint pattern, or RARA-PML expression status. The most striking differences were those between FLT3/ITD ${ }^{+}$samples and the rest. Sixty-four probe sets were up-regulated in the $\mathrm{ITD}^{+}$samples (Table S1), which included a number of genes with functions either known or postulated to be associated with cell growth and cell cycle control (eg, SOCS2, FRP1, PLAGL1, TTK, CDC16, APOBEC 3B) or with RNA processing (eg, GEMIN4, HNRPH1, DHX15). Conversely, 19 probe sets were down-regulated in $\mathrm{ITD}^{+}$samples (Table S2), of which 5 were
HLA class 1 genes (HLA-B71, allele $A * 2711, H L A-C w^{*} 1701$, $H L A-J, H L A-G 2.2)$. This analysis of a relatively small cohort of APL samples, therefore, suggests that the presence of ITD and D835 mutations have differing effects on gene expression in patients with a $\mathrm{t}(15 ; 17)$ abnormality and that both are distinct from expression patterns in FLT3 WT samples.

\section{In vitro response of APL blasts to ATRA and FLT3 inhibitor CEP-701}

Preliminary in vitro studies were performed using $P M L-R A R A^{+}$ NB4 cells, which had WT FLT3. In 3 independent experiments, incubation with $1 \mu \mathrm{M}$ ATRA for 2 or 3 days caused the cells to differentiate; the majority up-regulated CD11b, and approximately one third became $\mathrm{DCFDA}^{+}$, consistent with the acquisition of respiratory burst activity (Figure 3A). Incubation with $50 \mathrm{nM}$ CEP-701 did not cause apoptosis, with no change in the proportion of viable (annexin $\mathrm{V}^{-} / \mathrm{PI}^{-}$) cells, but did lead to a decrease in relative fold expansion (cell number) and absorbance in the MTS assay, suggesting inhibition of proliferation. Cells incubated with both ATRA and CEP-701 showed slightly higher levels of differentiation and a further decrease in the MTS assay.

These data suggested that adding an FLT3 inhibitor to standard doses of ATRA may be of benefit by causing growth inhibition of $P M L-R A R A^{+}$cells coupled to enhanced cell differentiation and maturation. Studies were, therefore, carried out using primary blast cells from $6 P M L-R A R A^{+}$patients. Four patients had ITDs; 3 had single mutants (relative levels $42 \%, 44 \%$, and $48 \%$, respectively), and 1 had 3 mutants (relative levels 23\%, 14\%, and 4\%). These results are consistent with a heterozygous mutation in most cells. Cells were assayed at day 4 or 5 , and representative results from one WT and one $\mathrm{ITD}^{+}$sample are shown in Figures 3B and 3C, respectively. Cells from all 6 patients showed an ATRA-induced response in the MTS assay (median, $176 \%$ of control; range, 133\%-314\%; Figure 4A) and up-regulated CD11b (median difference in percentage of $\mathrm{CD}_{11} \mathrm{~b}^{+}$between control cells and ATRAtreated cells, 26\%; range, $15 \%-72 \%$; Figure 4D), although the increase in functional maturation was more variable (median difference in percentage of $\mathrm{DCFDA}^{+}$between control cells and
A

Counts

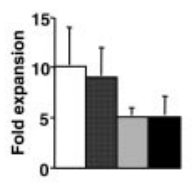

B
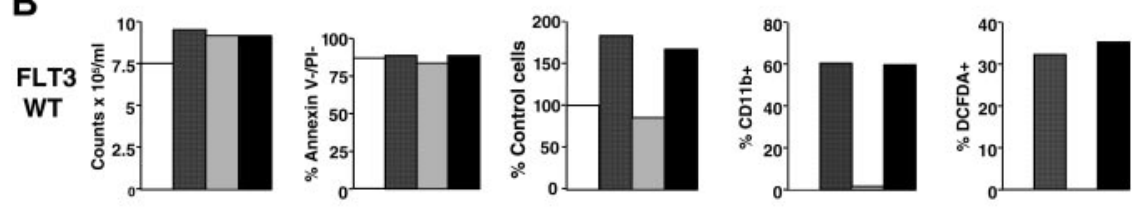

C

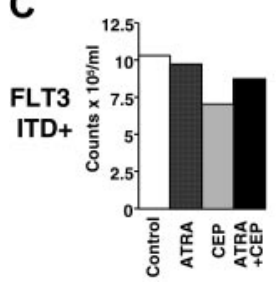

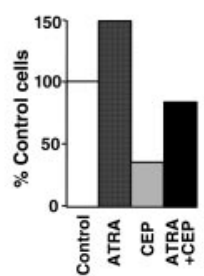

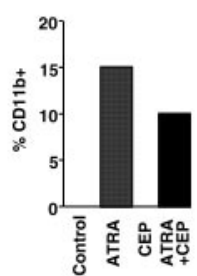

\% DCFDA+
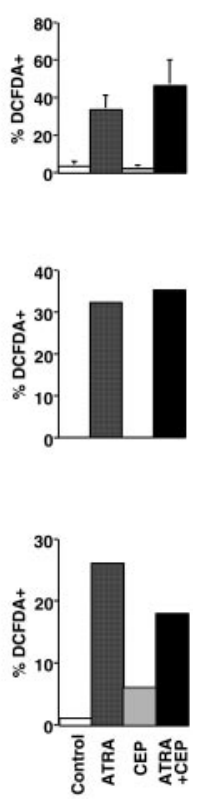

Figure 3. Effect of ATRA and CEP-701 on in vitro growth and differentiation of representative samples. Cells were cultured in RPMI/ $10 \%$ FCS for 3 to 5 days with either nothing further added (Control), $1 \mu \mathrm{M}$ ATRA, $50 \mathrm{nM}$ CEP-701, or $1 \mu \mathrm{M}$ ATRA $+50 \mathrm{nM}$ CEP-701, and then counted, used in an MTS assay, and immunophenotyped to determine the relative percentage of annexin $\mathrm{V}^{-} / \mathrm{PI}^{-}$, $\mathrm{CD}^{11 b^{+}}$, and DCFDA ${ }^{+}$cells. (A) NB4 cells. (B) Blasts from an FLT3 WT APL patient. (C) Blasts from an FLT3 ITD ${ }^{+}$APL patient. 
A
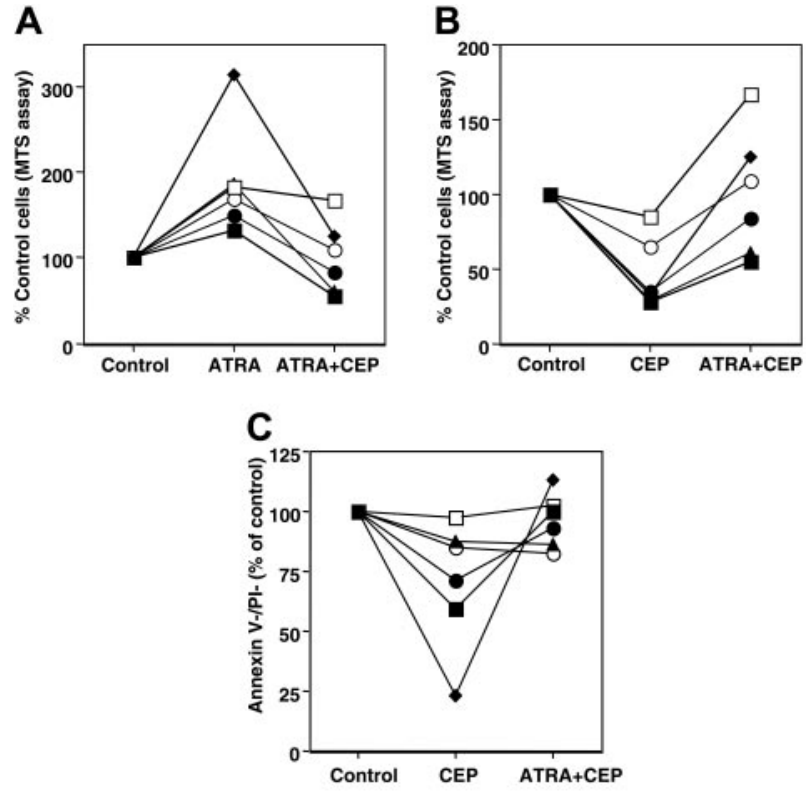

D

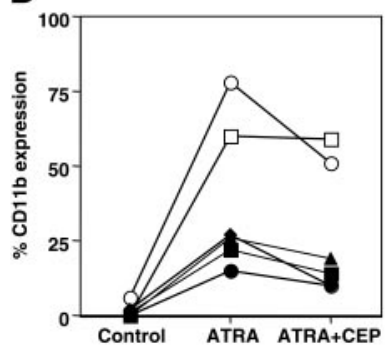

$\mathrm{E}$

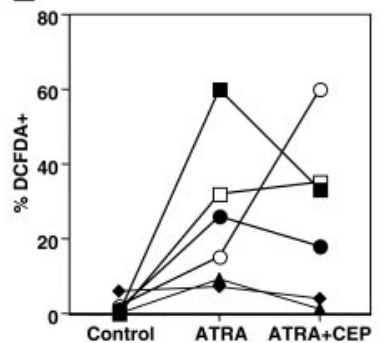

Figure 4. Response to ATRA and CEP-701 in blasts from 6 APL patients. Cells were cultured and assays performed as outlined in Figure 3. Two patients had WT FLT3 $(\square, \bigcirc)$, and 4 had ITD ${ }^{+}(\square, \bullet, \Delta, \bullet)$. Total mutant level was $41 \%$ to $48 \%$. One patient $(\boldsymbol{\Delta})$ had 3 mutants.

ATRA-treated cells, 19\%; range, 1\%-60\%; Figure 4E). Of note, the degree of CD11b up-regulation was considerably lower in the cells from the $4 \mathrm{ITD}^{+}$samples (relative increase in percentage of $\mathrm{CD}_{11} \mathrm{~b}^{+}$in ATRA-treated cells, 15\%-26\%) than in the $2 \mathrm{WT}$ samples $(60 \%$ and $72 \%)$ (Figure 4D).

There was a variable response to incubation with CEP-701. Cells from the 2 WT patients showed only a slight decrease in the MTS assay ( $85 \%$ and $65 \%$ of control) (Figure 4B) and little or no induction of apoptosis (annexin $\mathrm{V}^{-} / \mathrm{PI}^{-}$cells were $97 \%$ and $85 \%$, respectively, of that seen in control cells) (Figure 4C). Cells from the $4 \mathrm{ITD}^{+}$patients showed a marked decrease in the MTS assay in response to CEP-701 (28\%-35\% of control) with a variable reduction in viable cells (annexin $\mathrm{V}^{-} / \mathrm{PI}^{-}$cells $23 \%-87 \%$ of control), suggesting that at least some of this effect may be attributed to an increase in apoptosis.

When both agents were used together, the addition of CEP-701 significantly reduced the ATRA-induced increase in the MTS assay in most samples $(P=.015)$ (Figure 4A), particularly in the cells from the $\mathrm{ITD}^{+}$patients. If the values obtained using ATRA + CEP701 were compared with those using ATRA alone, the relative ATRA+CEP-701/ATRA ratios ranged between 0.3 and 0.6 in the 4 $\mathrm{ITD}^{+}$samples and was 0.6 and 0.9 in the 2 WT samples. However, in cells from all patients, irrespective of FLT3 status, the presence of ATRA significantly attenuated the effect of CEP-701 $(P=.004)$ (Figure 4B); the ATRA+CEP-701/CEP-701 ratio ranged between 1.7 and 3.8. In general, this correlated with a higher absolute cell

count and improved viability for the ATRA+CEP-701-treated cells (Figure 3B-C); the ratio of percentage of annexin $\mathrm{V}^{-} / \mathrm{PI}^{-}$cells for ATRA+CEP-701/CEP-701 ranged between 1.0 and 4.9 (Figure 4C). Adding CEP-701 also significantly decreased the level of ATRA-induced up-regulation of CD11b expression in all patients $(P=.039)$; the ATRA + CEP-701/ATRA ratio ranged between 0.32 and 0.98 (Figure 4D). Similar results were obtained with respect to functional maturation, with a decrease in the level of DCFDA positivity in $\mathrm{ITD}^{+}$but not WT cells in the presence of both agents (Figure 4E).

\section{Discussion}

Most patients with APL are now cured after first-line therapy with ATRA and anthracycline-based chemotherapy ${ }^{55}$; moreover, arsenic trioxide, and gemtuzumab ozogamicin (Mylotarg) have proven to be very effective targeted therapies for relapsed disease. ${ }^{56,57}$ However, to increase cure rates further, it would be helpful to rapidly identify patients with high-risk disease who could benefit from treatment modification. Multivariate analysis shows that WBC count is the most important prognostic factor in APL, and poorer outcomes in patients with high presenting WBC counts result from many factors, including higher rates of ID, particularly as a result of hemorrhage, higher incidence of retinoic acid syndrome, and increased RR. The basis for the high(er) WBC counts in patients with APL is not fully understood, but some studies have indicated that a significant proportion of such patients have FLT3 ITD. ${ }^{20-23,25,26}$ Results in our cohort of 203 patients are consistent with this; however, unlike other APL studies reported to date, we found that the presence of a D835/I836 mutation was also significantly associated with higher WBC count $(P=.001)$. Overall, $75 \%$ of our patients with a presenting WBC count of $10 \times 10^{9} / \mathrm{L}$ or greater had mutant FLT3. The presence of an FLT3 mutation correlated with a significantly higher rate of ID after the initiation of therapy $(P=.04)$. With the exception of one study of a smaller cohort of 42 patients, ${ }^{23}$ this impact of an FLT3 mutation on ID has not been found in other studies of APL patients. ${ }^{20,22,26}$ One possible explanation lies in the small number of patients with a D835/I836 mutation and their consequent amalgamation, in some studies, with patients with WT FLT3 into a single group for comparison of outcomes against those with ITDs. A further potential factor influencing ID rates in clinical studies relates to variation in the registration of patients with higher presenting WBC counts. Such patients are at very high risk for early fatal hemorrhage; therefore, patients with mutant FLT3 could be underrepresented if trial entry is delayed until $P M L-R A R A$ positivity is established. This may be less of a factor in the MRC studies, in which trial entry is based solely on a clinical suspicion of APL.

We were interested in establishing whether the presence of an FLT3 mutation was predictive of increased RR in APL, which could potentially influence postremission therapy and form a basis for rationalizing the frequency of minimal residual disease assessment according to risk. The current literature on the effect of FLT3 mutations on outcome in APL is conflicting. Some studies found no apparent difference in disease-free survival according to FLT3 status, ${ }^{17,21,23,25}$ whereas others reported a tendency toward, ${ }^{20,22}$ or significantly inferior, ${ }^{24}$ disease-free survival in patients with FLT3 ITDs. In the present study we found no evidence that the FLT3 mutation had an impact on RR, although the confidence interval was wide (Figure 1A, C). This raises the question of whether the impact of an ITD is different in APL than it is in other types of 
AML because in our previously reported study we found an ITD to be a highly significant and independent adverse prognostic factor for RR. ${ }^{13}$ Stratified analysis revealed no evidence of a difference between APL and non-APL patients treated within the UK MRC protocols with respect to the influence of FLT3 ITD status on their RR (Figure 1C), although again the possibility of a difference cannot be excluded given the wide confidence interval. This indicates that caution is required in interpreting the relapse data because of the limited numbers of patients involved. Nevertheless, when considered in the context of data from the other published cohorts, ${ }^{17,20-26}$ the results do suggest that the lesser impact of an ITD on relapse in APL is real. It was also possible that poorer outcomes for some of our patients resulted from the use of a short course of ATRA before induction chemotherapy, which was found to be worse for both $\mathrm{CR}$ and OS, but only in patients with presenting WBC counts less than $10 \times 10^{9} / \mathrm{L} .{ }^{45}$ However, we found no evidence that FLT3 status influenced outcome according to the timing of ATRA therapy. Further studies on larger cohorts of patients treated simultaneously with ATRA and induction chemotherapy are necessary to definitively determine the impact of an FLT3 mutation on outcome.

The high frequency of the FLT3 mutation in APL, particularly in patients whose WBC counts exceed $10 \times 10^{9} / \mathrm{L}$, has led to considerable interest in the potential clinical value of targeted therapy using FLT3 inhibitors in this disease. ${ }^{58}$ In vitro studies of cell lines have suggested that combining an FLT3 inhibitor with a differentiating agent may act synergistically to overcome the ITD-induced block in differentiation. ${ }^{30}$ Moreover, in a mouse model of FLT3 ITD $^{+}$APL, adding an FLT3 inhibitor to ATRA led to a significant increase in response rates and survival. ${ }^{37}$ Our preliminary studies in FLT3 WT NB4 cells were encouraging, and there was evidence of growth inhibition and enhanced differentiation in the presence of CEP-701 and ATRA. However, although CEP-701 was cytotoxic to primary PML-RARA ${ }^{+}$cells and this effect was greater in $\mathrm{ITD}^{+}$cells, the presence of ATRA attenuated the level of killing achieved, and the inhibitor also reduced the degree of ATRA-induced differentiation. These results introduce a note of caution for the up-front use of FLT3 inhibitors in patients with APL because diminution of the differentiation response could increase the risk for subsequent relapse. Furthermore, outcome results from our patients suggest that to be efficacious, FLT3 inhibitors would have to circumvent the early death rate if they are to lead to a significant improvement in overall outcome for APL patients. However, because half the deaths in this trial occurred within 4 days of trial entry, this goal may be unrealistic. Interestingly, a recent study has provided evidence for the presence of FLT3 ITDs in leukemic stem cells that may be responsive to FLT3 inhibitors. ${ }^{59}$ Although the nature of the APL stem cell remains poorly defined, ${ }^{3}$ the present study raises the possibility that FLT3 inhibitors could help to target therapy to this cell population in patients carrying a mutation; therefore, consideration should be given to the evaluation of FLT3 inhibitors as components of consolidation therapy.

The particular association of FLT3 mutation with the $t(15 ; 17)$ has led to interest in its relative contribution to the pathogenesis and biology of APL. The proliferative signal observed in vitro with both types of FLT3 mutation ${ }^{50}$ could readily account for the in vivo association with higher WBC count. Furthermore, our microarray analysis indicated the up-regulation of a number of genes associated with cell division in ITD $^{+}$samples, lending support to the hypothesis that specific mutations providing a proliferative/ survival signal cooperate with the PML-RARA-induced differen- tiation block in APL. ${ }^{8}$ In this regard it is interesting that the present study revealed an inverse relationship between frequency of FLT3/ITD and presence of additional cytogenetic abnormalities accompanying $\mathrm{t}(15 ; 17)$, suggesting that such genetic changes can substitute for the advantage provided by FLT3 mutation.

The observation that the presence of an ITD correlates with the expression of the reciprocal RARA-PML fusion transcript may provide insight into the underlying pathogenic mechanism(s) of $\mathrm{t}(15 ; 17)$ formation and FLT3 mutation. RARA-PML expression increases penetrance of the leukemic phenotype in PML-RARA transgenic mice $^{7}$ and has been thought to increase genomic instability, which is in keeping with the aberrant DNA duplication of an ITD. Its expression is also consistent with the recent proposal that DNA topoisomerase II may be implicated in the DNA damage process, leading to the formation of $\mathrm{t}(15 ; 17)$ and the mutation of FLT3. ${ }^{60}$ Although involvement of this enzyme in the etiology of de novo disease is subject to conjecture, we have recently demonstrated that it mediates DNA damage leading to the formation of the chromosomal translocation in therapy-related APL, ${ }^{61}$ and such clean breakage and re-ligation of DNA may well be involved in ITD generation. It is also noteworthy that there are striking differences in the relative incidence of FLT3 ITDs and point mutations between APL patients in Europe/North America and Asia ${ }^{13-26,50}$ and a significantly varied propensity for the disease to develop in different ethnic groups, ${ }^{62}$ suggesting that genetic or environmental influences may play a role in the pathogenesis of APL.

Of biologic interest is the observation that morphologic features and PML breakpoint distribution of patients with an FLT3 ITD differed from those with a D835/I836 mutation or with WT FLT3 (Table 1). The reasons for these intriguing correlations are unclear, but it is possible that the nature of the hematopoietic progenitor that is the cellular target in APL has a critical influence, both in terms of the genetic lesions required for leukemic transformation and in the disease characteristics, including morphologic features. ${ }^{3}$ Although PML-RARA expression in myeloid progenitors leads to the accumulation of hypergranular blasts in transgenic mice, ${ }^{63}$ coexpression of FLT3 ITD yields a disease more reminiscent of M3v with hypogranular blasts with lobulated nuclei. ${ }^{37}$ This suggests that the ITD can contribute to the generation of morphologic features of M3v.

Overall, our study not only highlights the prevalence of FLT3 mutation in patients with APL and the poorer prognosis because of the high risk for induction death, it reveals correlations with a number of molecular features that may be pertinent to the cellular origins and processes necessary for leukemic transformation. It will be interesting to establish the range of progenitors permissive for transformation by the $t(15 ; 17)$ and the different spectra of cooperating lesions required for the leukemic phenotype in each cellular context.

\section{Acknowledgments}

We thank all the clinical investigators who entered and managed patients in these trials, the data managers who provided clinical data, the staff who performed cytogenetic analyses, and $\mathrm{Si}$ vartharsini Srirangan and Yashma Patel for technical assistance. We are particularly indebted to Dr Sally Killick, Dr Mary Taj, Prof Richard Clark, and Prof John Reilly for providing diagnostic material for in vitro assays. 


\section{Appendix}

Following is a complete list of members of the NCRI Adult Leukaemia Working Party: Dr S. Ali, Hull Royal Infirmary; Prof J. F. Apperley, Imperial College School of Medicine, London; Dr D. T. Bowen, Ninewells Hospital, Dundee; Dr P. V. Browne, St. James' Hospital, Dublin, Republic of Ireland; Prof. A. K. Burnett (chairman), University of Wales College of Medicine, Cardiff; Prof D. Catovsky, Institute of Cancer Research, Sutton; Dr J. D. Cavenagh, The Royal London Hospital; Prof J. A. Child, Leeds General Infirmary; Prof. R. E. Clark, Royal Liverpool Hospital, Liverpool; Prof C. F. Craddock, Queen Elizabeth Medical Centre, Birmingham; Dr D. J. Culligan, Aberdeen Royal Infirmary; Dr R. J. G. Cuthbert, Belfast City Hospital; Dr C. E. Dearden, St George's Hospital Medical School, London; Prof. J. M. Goldman, Imperial College School of Medicine, London; Prof A. H. Goldstone, University College London Hospitals; Prof A. R. Green, University of Cambridge; Dr D. J. Grimwade, King's College London; Prof T. J. Hamblin, Royal Bournemouth Hospital;
Dr C. N. Harrison, St Thomas' Hospital, London; Prof P. Hillmen, University of Leeds; Dr R. K. Hills, University of Birmingham Clinical Trials Unit, Edgbaston, Birmingham; Dr A. E. Hunter, Leicester Royal Infirmary NHS Trust, Leicester; Dr G. H. Jackson, Royal Victoria Infirmary, Newcastle upon Tyne; Dr T. J. Littlewood, John Radcliffe Hospital, Oxford; Prof S. MacKinnon, Royal Free Hospital, London; Dr M. F. McMullin, Belfast City Hospital; Prof D. W. Milligan, Heartlands Hospital, Birmingham; Prof. G. J. Morgan, Royal Marsden Hospital, Sutton; Prof A. C. Newland, The Royal London Hospital; Dr S. G. O’Brien, Royal Victoria Infirmary, Newcastle upon Tyne; Dr A. Peniket, John Radcliffe Hospital, Oxford; Prof A. G. Prentice, Plymouth General Hospital; Prof S. J. Proctor, Royal Victoria Infirmary, Newcastle upon Tyne; Dr S. Richards, Clinical Trials Service Unit, Radcliffe Infirmary, Oxford; Dr D. S. Richardson, Southampton General Hospital; Prof N. H. Russell, City Hospital, Nottingham; Dr S. A. Schey, King's College Hospital, London; Dr C. R. J. Singer, Royal United Hospital, Bath; Dr P. J. Tansey, Victoria Infirmary, Glasgow; Prof K. Wheatley, University of Birmingham Clinical Trials Unit, Edgbaston, Birmingham; Prof J. A. L. Yin, Manchester Royal Infirmary.

\section{References}

1. Mistry AR, Pedersen EW, Solomon E, Grimwade $D$. The molecular pathogenesis of acute promyelocytic leukaemia: implications for the clinical management of the disease. Blood Rev. 2003;17: 71-97.

2. Westervelt P, Ley TJ. Seed versus soil: the importance of the target cell for transgenic models of human leukemias. Blood. 1999;93:2143-2148.

3. Grimwade D, Enver T. Acute promyelocytic leukemia: where does it stem from? Leukemia. 2004; 18:375-384

4. Alcalay M, Zangrilli D, Fagioli M, et al. Expression pattern of the RAR alpha-PML fusion gene in acute promyelocytic leukemia. Proc Natl Acad Sci U S A. 1992;89:4840-4844.

5. Borrow J, Goddard AD, Gibbons B, et al. Diagnosis of acute promyelocytic leukaemia by RT-PCR: detection of PML-RARA and RARA-PML fusion transcripts. Br J Haematol. 1992;82:529-540.

6. Grimwade D, Howe K, Langabeer S, et al. Establishing the presence of the $t(15 ; 17)$ in suspected acute promyelocytic leukaemia: cytogenetic, molecular and PML immunofluorescence assessment of patients entered into the M.R.C. ATRA trial. M.R.C. Adult Leukaemia Working Party. Br J Haematol. 1996;94:557-573.

7. Pollock JL, Westervelt P, Kurichety AK, Pelicci PG, Grisolano JL, Ley TJ. A bcr-3 isoform of RARalpha-PML potentiates the development of PML-RARalpha-driven acute promyelocytic leukemia. Proc Natl Acad Sci U S A. 1999;96:1510315108.

8. Deguchi K, Gilliland DG. Cooperativity between mutations in tyrosine kinases and in hematopoietic transcription factors in AML. Leukemia. 2002; 16:740-744.

9. Gilliland DG, Griffin JD. The roles of FLT3 in hematopoiesis and leukemia. Blood. 2002;100: 1532-1542.

10. Kottaridis PD, Gale RE, Linch DC. Flt3 mutations and leukaemia. Br J Haematol. 2003;122:523538

11. Stirewalt DL, Radich JP. The role of FLT3 in haematopoietic malignancies. Nat Rev Cancer. 2003:3:650-665.

12. Levis M, Small D. FLT3: ITDoes matter in leukemia. Leukemia. 2003;17:1738-1752

13. Kottaridis PD, Gale RE, Frew ME, et al. The presence of a FLT3 internal tandem duplication in patients with acute myeloid leukemia (AML) adds important prognostic information to cytogenetic risk group and response to the first cycle of chemotherapy: analysis of 854 patients from the United Kingdom Medical Research Council AML 10 and 12 trials. Blood. 2001;98:1752-1759.
14. Thiede C, Steudel C, Mohr B, et al. Analysis of FLT3-activating mutations in 979 patients with acute myelogenous leukemia: association with FAB subtypes and identification of subgroups with poor prognosis. Blood. 2002;99:4326-4335.

15. Schnittger S, Schoch C, Dugas M, et al. Analysis of FLT3 length mutations in 1003 patients with acute myeloid leukemia: correlation to cytogenetics, FAB subtype, and prognosis in the AMLCG study and usefulness as a marker for the detection of minimal residual disease. Blood. 2002; 100:59-66.

16. Frohling S, Schlenk RF, Breitruck J, et al. Prognostic significance of activating FLT3 mutations in younger adults (16 to 60 years) with acute myeloid leukemia and normal cytogenetics: a study of the AML Study Group Ulm. Blood. 2002;100: 4372-4380.

17. Kainz B, Heintel D, Marculescu R, et al. Variable prognostic value of FLT3 internal tandem duplications in patients with de novo AML and a normal karyotype, $t(15 ; 17), t(8 ; 21)$ or inv(16). Hematol J. 2002;3:283-289

18. Moreno I, Martin G, Bolufer P, et al. Incidence and prognostic value of FLT3 internal tandem duplication and D835 mutations in acute myeloid leukemia. Haematologica. 2003;88:19-24.

19. Chillon MC, Fernandez C, Garcia-Sanz R, et al. FLT3-activating mutations are associated with poor prognostic features in AML at diagnosis but they are not an independent prognostic factor. Hematol J. 2004;5:239-246.

20. Noguera NI, Breccia M, Divona M, et al. Alterations of the FLT3 gene in acute promyelocytic leukemia: association with diagnostic characteristics and analysis of clinical outcome in patients treated with the Italian AIDA protocol. Leukemia. 2002;16:2185-2189.

21. Shih LY, Kuo MC, Liang DC, et al. Internal tandem duplication and Asp835 mutations of the FMS-like tyrosine kinase 3 (FLT3) gene in acute promyelocytic leukemia. Cancer. 2003;98:12061216.

22. Au WY, Fung A, Chim CS, et al. FLT-3 aberrations in acute promyelocytic leukaemia: clinicopathological associations and prognostic impact. $\mathrm{Br} \mathrm{J}$ Haematol. 2004;125:463-469.

23. Palumbo GA, Tanteri V, Buglio D, et al. FLT3-activating mutations in acute promyelocytic leukemia: higher incidence of ITD in BCR3 patients [abstract]. Blood. 2002;100:326a.

24. Gallagher RE, Lee SJ, Sankoorikal B-J, Wiernik $\mathrm{PH}$, Tallman MS. Independent prognostic significance of FLT3 internal tandem duplication (ITD) mutations in acute promyelocytic leukemia (APL) patients treated on ECOG protocol E2491 [abstract]. Blood. 2002;100:326a.
25. Kiyoi H, Naoe T, Yokota S, et al. Internal tandem duplication of FLT3 associated with leukocytosis in acute promyelocytic leukemia: Leukemia Study Group of the Ministry of Health and Welfare (Kohseisho). Leukemia. 1997;11:1447-1452.

26. Callens C, Chevret S, Cayuela JM, et al. Prognostic implication of FLT3 and Ras gene mutations in patients with acute promyelocytic leukemia (APL): a retrospective study from the European APL Group. Leukemia. 2005;19,11531160.

27. Levis M, Tse KF, Smith BD, Garrett E, Small D. A FLT3 tyrosine kinase inhibitor is selectively cytotoxic to acute myeloid leukemia blasts harboring FLT3 internal tandem duplication mutations. Blood. 2001;98:885-887.

28. Levis M, Allebach J, Tse KF, et al. A FLT3-targeted tyrosine kinase inhibitor is cytotoxic to leukemia cells in vitro and in vivo. Blood. 2002;99:38853891.

29. Tse KF, Allebach J, Levis M, Smith BD, Bohmer FD, Small D. Inhibition of the transforming activity of FLT3 internal tandem duplication mutants from AML patients by a tyrosine kinase inhibitor. Leukemia. 2002;16:2027-2036.

30. Zheng R, Friedman AD, Small D. Targeted inhibition of FLT3 overcomes the block to myeloid differentiation in 32Dcl3 cells caused by expression of FLT3/ITD mutations. Blood. 2002;100:41544161.

31. Zheng R, Friedman AD, Levis M, Li L, Weir EG, Small D. Internal tandem duplication mutation of FLT3 blocks myeloid differentiation through suppression of C/EBPalpha expression. Blood. 2004; 103:1883-1890.

32. Brown P, Meshinchi S, Levis M, et al. Pediatric AML primary samples with FLT3/ITD mutations are preferentially killed by FLT3 inhibition. Blood. 2004; 104:1841-1849.

33. Griswold IJ, Shen LJ, La Rosee P, et al. Effects of MLN518, a dual FLT3 and KIT inhibitor, on normal and malignant hematopoiesis. Blood. 2004;104: 2912-2918.

34. Yee KW, Schittenhelm M, O'Farrell AM, et al. Synergistic effect of SU11248 with cytarabine or daunorubicin on FLT3 ITD-positive leukemic cells. Blood. 2004;104:4202-4209.

35. Kelly LM, Yu JC, Boulton CL, et al. CT53518, a novel selective FLT3 antagonist for the treatment of acute myelogenous leukemia (AML). Cancer Cell. 2002;1:421-432.

36. Weisberg E, Boulton C, Kelly LM, et al. Inhibition of mutant FLT3 receptors in leukemia cells by the small molecule tyrosine kinase inhibitor PKC412. Cancer Cell. 2002;1:433-443.

37. Sohal J, Phan VT, Chan PV, et al. A model of APL 
with FLT3 mutation is responsive to retinoic acid and a receptor tyrosine kinase inhibitor, SU11657. Blood. 2003;101:3188-3197.

38. O'Farrell AM, Abrams TJ, Yuen HA, et al. SU11248 is a novel FLT3 tyrosine kinase inhibitor with potent activity in vitro and in vivo. Blood. 2003;101:3597-3605.

39. Giles FJ, Stopeck AT, Silverman LR, et al. SU5416, a small molecule tyrosine kinase receptor inhibitor, has biologic activity in patients with refractory acute myeloid leukemia or myelodysplastic syndromes. Blood. 2003;102:795-801.

40. Fiedler $\mathrm{W}$, Mesters $\mathrm{R}$, Tinnefeld $\mathrm{H}$, et al. A phase 2 clinical study of SU5416 in patients with refractory acute myeloid leukemia. Blood. 2003;102: 2763-2767.

41. Fiedler W, Serve H, Dohner H, et al. A phase I study of SU11248 in the treatment of patients with refractory or resistant acute myeloid leukemia (AML) or not amenable to conventional therapy for the disease. Blood. 2005;105:986-993.

42. O'Farrell AM, Foran JM, Fiedler W, et al. An innovative phase I clinical study demonstrates inhibition of FLT3 phosphorylation by SU11248 in acute myeloid leukemia patients. Clin Cancer Res. 2003;9:5465-5476.

43. Smith BD, Levis M, Beran M, et al. Single-agent CEP-701, a novel FLT3 inhibitor, shows biologic and clinical activity in patients with relapsed or refractory acute myeloid leukemia. Blood. 2004; 103:3669-3676

44. Stone RM, Deangelo DJ, Klimek V, et al. Patients with acute myeloid leukemia and an activating mutation in FLT3 respond to a small-molecule FLT3 tyrosine kinase inhibitor, PKC412. Blood. 2005; 105:54-60.

45. Burnett AK, Grimwade D, Solomon E, Wheatley $\mathrm{K}$, Goldstone AH. Presenting white blood cell count and kinetics of molecular remission predict prognosis in acute promyelocytic leukemia treated with all-trans retinoic acid: result of the Randomized MRC Trial. Blood. 1999;93:4131 4143
46. Grimwade D, Walker H, Oliver F, et al. The importance of diagnostic cytogenetics on outcome in AML: analysis of 1,612 patients entered into the MRC AML 10 trial: the Medical Research Council Adult and Children's Leukaemia Working Parties. Blood. 1998;92:2322-2333.

47. Bennett JM, Catovsky D, Daniel MT, et al. Proposed revised criteria for the classification of acute myeloid leukemia: a report of the FrenchAmerican-British Cooperative Group. Ann Intern Med. 1985;103:620-625

48. McKenna RW, Parkin J, Bloomfield CD, Sundberg RD, Brunning RD. Acute promyelocytic leukaemia: a study of 39 cases with identification of a hyperbasophilic microgranular variant. $\mathrm{Br} \mathrm{J}$ Haematol. 1982;50:201-214.

49. Sainty D, Liso V, Cantu-Rajnoldi A, et al. A new morphologic classification system for acute promyelocytic leukemia distinguishes cases with underlying PLZF/RARA gene rearrangements: Group Francais de Cytogenetique Hematologique, UK Cancer Cytogenetics Group and BIOMED 1 European Community-Concerted Action "Molecular Cytogenetic Diagnosis in Haematological Malignancies." Blood. 2000;96:12871296.

50. Yamamoto $\mathrm{Y}$, Kiyoi $\mathrm{H}$, Nakano $\mathrm{Y}$, et al. Activating mutation of D835 within the activation loop of FLT3 in human hematologic malignancies. Blood. 2001;97:2434-2439.

51. Tusher VG, Tibshirani R, Chu G. Significance analysis of microarrays applied to the ionizing radiation response. Proc Natl Acad Sci U S A. 2001:98:5116-5121

52. Lanotte M, Martin-Thouvenin V, Najman S, Balerini P, Valensi F, Berger R. NB4, a maturation inducible cell line with $t(15 ; 17)$ marker isolated from a human acute promyelocytic leukemia (M3). Blood. 1991;77:1080-1086.

53. Jaswon MS, Khwaja A, Roberts PJ, Jones HM, Linch DC. The effects of rhGM-CSF on the neutrophil respiratory burst when studied in whole blood. Br J Haematol. 1990;75:181-187.
54. Cheson BD, Cassileth PA, Head DR, et al. Report of the National Cancer Institute-sponsored workshop on definitions of diagnosis and response in acute myeloid leukemia. J Clin Oncol. 1990;8: 813-819.

55. Tallman MS, Rowe JM. Long-term follow-up and potential for cure in acute promyelocytic leukaemia. Best Pract Res Clin Haematol. 2003;16:535543.

56. Douer D, Tallman MS. Arsenic trioxide: new clinical experience with an old medication in hematologic malignancies. J Clin Oncol. 2005;23:23962410

57. Lo-Coco F, Cimino G, Breccia M, et al. Gemtuzumab ozogamicin (Mylotarg) as a single agent for molecularly relapsed acute promyelocytic leukemia. Blood. 2004;104:1995-1999.

58. Gilliland DG. FLT3-activating mutations in acute promyelocytic leukaemia: a rationale for riskadapted therapy with FLT3 inhibitors. Best Pract Res Clin Haematol. 2003;16:409-417.

59. Levis M, Murphy KM, Pham R et al. Internal tandem duplications of the FLT3 gene are present in leukemia stem cells. Blood. 2005; 106 :673-680.

60. Libura M, Asnafi V, Tu A, et al. FLT3 and MLL intragenic abnormalities in AML reflect a common category of genotoxic stress. Blood. 2003;102: 2198-2204.

61. Mistry AR, Felix CA, Whitmarsh RJ, et al. DNA topoisomerase II in therapy-related acute promyelocytic leukemia. N Engl J Med. 2005;352:15291538

62. Douer D, Santillana S, Ramezani L, et al. Acute promyelocytic leukaemia in patients originating in Latin America is associated with an increased frequency of the bcr1 subtype of the PML/RARalpha fusion gene. Br J Haematol. 2003;122:563570.

63. Brown D, Kogan S, Lagasse E, et al. A PMLRARalpha transgene initiates murine acute promyelocytic leukemia. Proc Natl Acad Sci U S A. 1997;94:2551-2556. 